九州大学学術情報リポジトリ

Kyushu University Institutional Repository

Introducing Viewpoints of Mechanics into Basic Growth Analysis: (X) Wave Functions in Comparison and Relationship with Growth Functions

Shimojo, Masataka

Laboratory of Animal Feed Science, Division of Animal Science, Department of Animal and Marine Bioresource Sciences, Faculty of Agriculture, Kyushu University

https://doi.org/10.5109/14050

出版情報：九州大学大学院農学研究院紀要. 54 (1)，pp.141-145，2009-02-27. Faculty of Agriculture, Kyushu University

バージョン：

権利関係 : 


\title{
Introducing Viewpoints of Mechanics into Basic Growth Analysis (X) Wave Functions in Comparison and Relationship with Growth Functions -
}

\author{
Masataka SHIMOJO* \\ Laboratory of Animal Feed Science, Division of Animal Science, Department of Animal \\ and Marine Bioresource Sciences, Faculty of Agriculture, Kyushu University, \\ Fukuoka 812-8581, Japan \\ (Received November 14, 2008 and accepted December 5, 2008)
}

\begin{abstract}
This study was conducted to investigate wave functions derived from introducing growth jerk into basic growth mechanics by paying attention to the comparison and relationship with growth functions. Positive and negative signs, which appeared in taking the square root when solving the differential equation, gave wave functions $\left[\Psi=\left( \pm W_{0}\right) \cdot( \pm \exp (( \pm \boldsymbol{i} R G R) \cdot t))\right]$ as well as growth functions $\left[W=\left( \pm W_{0}\right) \cdot( \pm \exp (( \pm \mathrm{RGR})\right.$ $\cdot t))$. The results obtained were as follows. (1) Growth functions suggested basic phenomena of ruminant agriculture through the concept of energy conservation and an open system adopted by living things that showed degradation and synthesis of body components. (2) Wave functions gave anticlockwise and clockwise rotations. Three-dimensional coordinates suggested from wave functions gave right-handed spirals, and left-handed spirals were derived when some wave functions were given another way of description. (3) Wave functions were related with growth functions through hypothetic operations bridging the weight gap between $0 \pm \boldsymbol{i} W_{\mathbf{0}}$ and $\pm W_{\mathbf{0}}$. It was suggested that introducing growth jerk into basic growth mechanics gave some pieces of information about properties of wave functions in the comparison and relationship with growth functions.
\end{abstract}

\section{INTRODUCTION}

My previous two reports (Shimojo, 2006, 2007) suggested that introducing growth jerk (the rate of change of growth acceleration) into basic growth mechanics led to a differential equation. Solving this differential equation suggested wave functions as well as gave growth functions for the ruminant animal and the forage plant. What wave functions mean is not known and they are absolutely different from growth functions. However, wave and growth functions were derived from manipulations that conserved differential rules against giving positive and negative signs to each of the terms when taking the square root of them (Shimojo, 2006, 2007). However, those two reports include mistakes, misunderstanding, lack of explanation and insufficient discussion. It is very difficult to compare wave functions with growth functions, because the former are described using complex numbers and the latter are described using real numbers. There is a great wall or gap between them that is very difficult to get over or bridge.

The present study was designed to investigate properties of wave functions derived from introducing growth jerk into basic growth mechanics by paying attention to the comparison and relationship with growth functions.

\section{PROPERTIES OF WAVE FUNCTIONS IN COMPARISON AND RELATIONSHIP WITH GROWTH FUNCTIONS}

\section{(A) Introducing growth jerk into basic growth}

\footnotetext{
* Corresponding Author (E-mail: mshimojo@agr.kyushu-u. ac.jp)
}

\section{mechanics}

Introducing growth jerk into basic growth mechanics is given by a series of the following calculations.

$$
W=W_{0} \cdot \exp (\mathrm{RGR} \cdot t)
$$

where $W=$ weight, $t=$ time, $\mathrm{RGR}=$ relative growth rate, $W_{\mathbf{0}}=$ the weight at $t=0$.

A series of differential calculations is as follows,

$$
\begin{aligned}
& d W / d t=\mathrm{AGR}=\mathrm{RGR} \cdot W_{0} \cdot \exp (\mathrm{RGR} \cdot t), \\
& d^{2} W / d t^{2}=\mathrm{GA}=\mathrm{RGR}^{2} \cdot W_{0} \cdot \exp (\mathrm{RGR} \cdot t), \\
& d^{3} W / d t^{3}=\mathrm{GJ}=\mathrm{RGR}^{3} \cdot W_{0} \cdot \exp (\mathrm{RGR} \cdot t),
\end{aligned}
$$

where AGR=absolute growth rate, GA=growth acceleration, GJ=growth jerk.

$$
\begin{aligned}
& \frac{d^{2} W / d t^{2}}{d W / d t}=\frac{d^{3} W / d t^{3}}{d^{2} W / d t^{2}}=\mathrm{RGR}, \\
& \left(d^{2} W / d t^{2}\right)^{2}=(d W / d t) \cdot\left(d^{3} W / d t^{3}\right)
\end{aligned}
$$

The square root of function (6) is given by

$$
\begin{aligned}
& d^{2} W / d t^{2}= \pm \sqrt{(d W / d t) \cdot\left(d^{3} W / d t^{3}\right)} \\
& = \pm \sqrt{\left(\mathrm{RGR} \cdot W_{0} \cdot \exp (\mathrm{RGR} \cdot t)\right) \cdot\left(\mathrm{RGR}^{3} \cdot W_{0} \cdot \exp (\mathrm{RGR} \cdot t)\right)} \\
& = \pm \sqrt{W_{0}^{2} \cdot \mathrm{RGR}^{4} \cdot(\exp (\mathrm{RGR} \cdot t))^{2}} \\
& = \pm\left(W_{0}\right) \cdot\left(\mathrm{RGR}^{2}\right) \cdot(\exp (\mathrm{RGR} \cdot t)) .
\end{aligned}
$$




\section{(B) Giving positive and negative signs to each of three terms in function (7)}

In this report, I will give positive and negative signs $( \pm)$ to each of the three terms in the right-hand side of function (7) from the viewpoint of mathematical interests. There occurs a split into the function with real numbers and that with complex numbers when \pm signs are given to the second term (Shimojo, 2006, 2007). Therefore, this operation is taken up first.

Signs $( \pm)$ to the second term is given by

$$
d^{2} W / d t^{2}=\left(W_{0}\right) \cdot\left( \pm \mathrm{RGR}^{2}\right) \cdot(\exp (\mathrm{RGR} \cdot t)) .
$$

The function with real numbers is given by

$$
d^{2} W / d t^{2}=\left(W_{0}\right) \cdot\left(\mathrm{RGR}^{2}\right) \cdot(\exp (\mathrm{RGR} \cdot t)) .
$$

Since $\mathrm{RGR}^{2}$ in function (9) leads to function (10), function (1) is replaced with function (11) in order to conserve differential rules,

$$
\begin{aligned}
& \sqrt{\mathrm{RGR}^{2}}= \pm \mathrm{RGR}, \\
& W=W_{0} \cdot \exp (( \pm \mathrm{RGR}) \cdot t) .
\end{aligned}
$$

The function with complex numbers is given by

$$
d^{2} W / d t^{2}=\left(W_{0}\right) \cdot\left(-\mathrm{RGR}^{2}\right) \cdot(\exp (\mathrm{RGR} \cdot t))
$$

Since $-R_{G R}^{2}$ in function (12) leads to function (13), growth function (1) is replaced with wave function (14) in order to conserve differential rules,

$$
\begin{aligned}
& \sqrt{-\mathrm{RGR}^{2}}= \pm \boldsymbol{i} \mathrm{RGR}, \\
& \Psi=W_{0} \cdot \exp (( \pm \boldsymbol{i} \mathrm{RGR}) \cdot t),
\end{aligned}
$$

where $\boldsymbol{i}=$ imaginary unit, $\Psi=$ wave function.

Signs $( \pm)$ to the first term is given by

$$
d^{2} W / d t^{2}=\left( \pm W_{0}\right) \cdot\left(\mathrm{RGR}^{2}\right) \cdot(\exp (\mathrm{RGR} \cdot t)) .
$$

Conserving differential rules requires replacing function (1) with function (16),

$$
W=\left( \pm W_{\mathbf{0}}\right) \cdot \exp (\mathrm{RGR} \cdot t)
$$

Signs $( \pm)$ to the third term is given by

$$
d^{2} W / d t^{2}=\left(W_{0}\right) \cdot\left(\mathrm{RGR}^{2}\right) \cdot( \pm \exp (\mathrm{RGR} \cdot t)) .
$$

Conserving differential rules requires replacing function (1) with function (18),

$$
W=W_{0} \cdot( \pm \exp (\mathrm{RGR} \cdot t))
$$

Inserting functions (16) and (18) into each of functions (11) and (14) gives growth function (19) and wave function (20),

$$
\begin{aligned}
& W=\left( \pm W_{0}\right) \cdot( \pm \exp (( \pm \mathrm{RGR}) \cdot t)), \\
& \Psi=\left( \pm W_{0}\right) \cdot( \pm \exp (( \pm \boldsymbol{i} \mathrm{RGR}) \cdot t)) .
\end{aligned}
$$

(C) Growth function, wave function and comparing them

(C-1) Growth Function (19)

Growth function (19) gives eight functions by the combination of three sets of \pm signs. Shimojo et al. (2008) tried to relate properties of eight functions with phenomena of ruminant agriculture using differential equation (21) that is given by relating functions (1) (3),

$$
(d W / d t)^{2}=W \cdot\left(d^{2} W / d t^{2}\right),
$$

Here again, I will relate function (19) to ruminant agriculture (Shimojo et al., 2008) with some modification and addition. This is briefly shown in (i) (iv). (i) The positive weight $\left(W_{0}\right)$ and negative weight $\left(-W_{0}\right)$ suggest a kind of energy conservation between depriving and being deprived. The negative sign of $-W_{\mathbf{0}}$ is removed if there is a translation of coordinate axes. When $W_{\mathbf{0}}$ is the ruminant body weight, $-W_{\mathbf{0}}$ is interpreted as the weight of forage feed expressed as $W_{\mathbf{0}}$. The actual weight of forage feed is described using the product of $W_{\mathbf{0}}$ and feed conversion ratio. This might give a mathematical explanation to the fact that living things adopt an open system. (ii) There is not only synthesis (RGR) but also degradation (-RGR) of body components for both the ruminant animal and the forage plant. There is an increase in body weight when synthesis exceeds degradation, but body weight decreases when degradation exceeds synthesis. The body weight is kept constant by the equality between degradation and synthesis. (iii) The term $\exp (\mathrm{RGR} \cdot t)$ does not change the form of function, but $-\exp (\mathrm{RGR} \cdot t)$ changes $W_{\mathbf{0}}$ into $-W_{0}$ and $-W_{0}$ into $W_{0}$. This suggests that components of the ruminant body are originally reduced to the forage feed and the forage feed is transformed into the ruminant body. (iv) If time $(t)$ in $W=W_{0} \cdot \exp (\mathrm{RGR} \cdot t)$ is given \pm signs, then the negative sign of $-t$ is absorbed by RGR to become $-\mathrm{RGR}$ and recovering to RGR requires an operation: $-\mathrm{RGR}+2 \mathrm{RGR}=\mathrm{RGR}$. This might remind me of degradation and synthesis in body components. Does this suggest the regulation of the increase in entropy? (v) Ruminant-forage relationships might be related to conserving differential rules against mathematical operations that are used to solve differential equations (19) and (21). Might this be a kind of mystery that connects abstract concepts of mathematics with concrete phenomena of ruminant agriculture?

Growth function (19) shows that $W$ and $-W$ take values: $-W \leq-W_{0},-W_{0} \leq-W<0,0<W \leq W_{0}, W_{0} \leq$ $W$. The feature of growth function (19) is that $W$ does not take the value of zero owing to its mathematical properties and corresponding differential equation (22) where the denominator is composed of $W$ in the first term of the left-hand side, 
$(1 / W) \cdot(d W / d t)=\mathrm{RGR}$.

Basic growth function requires $W$ that is not zero at the starting point, in other words, nothing comes from nothing. There is a weight gap between 0 and $W$ and between 0 and $-W$. Boldly speaking at the risk of making mistakes, are there any operations that bridge this weight gap?

(C-2) Wave Function (20)

It is known from Euler's formula that wave function (20) is described using trigonometric functions (20-1) that show wave properties clearly,

$$
\begin{aligned}
\Psi & =\left( \pm W_{0}\right) \cdot( \pm \exp (( \pm \boldsymbol{i} R G R) \cdot t)) \\
& =\left( \pm W_{0}\right) \cdot( \pm(\cos (\mathrm{RGR} \cdot t) \pm \boldsymbol{i} \sin (\mathrm{RGR} \cdot t)))
\end{aligned}
$$

Wave function (20-1) takes values between $-W_{\mathbf{0}}+\boldsymbol{i} 0$ and $W_{0}+\boldsymbol{i} 0$ and between $0-\boldsymbol{i} W_{0}$ and $0+\boldsymbol{i} W_{0}$ on the complex plane. The feature of wave function (20-1) is that the real part of it takes the value of zero in the form of $0-\boldsymbol{i} W_{\mathbf{0}}$ and $0+\boldsymbol{i} W_{\mathbf{0}}$. Does this suggest in wave functions (20) and (21) that there is not the state of absolute zero because of the existence of imaginary numbers?

(C-3) Relationship between wave function (20) and growth function (19) in the weight gap

Boldly speaking at the risk of making mistakes, if there is a simple try to bridge the weight gap between 0 and $\pm W$, then wave function (20) might be related with growth function (19) through the following transformation,

$$
\begin{aligned}
& \Psi=\left( \pm W_{0}\right) \cdot( \pm \exp (( \pm \boldsymbol{i} \mathrm{RGR}) \cdot t)) \\
& \rightarrow\left( \pm W_{0}\right) \cdot( \pm \exp (( \pm \boldsymbol{i}) \cdot( \pm \boldsymbol{i} \mathrm{RGR}) \cdot t)) \\
& =\left( \pm W_{0}\right) \cdot( \pm \exp (( \pm \mathrm{RGR}) \cdot t))=W .
\end{aligned}
$$

This transformation suggests that wave function (20) exists between the two growth functions (19-1) and (19-2), a kind of mid-existence,

$$
\begin{aligned}
& W=\left( \pm W_{\mathbf{0}}\right) \cdot( \pm \exp ((\mathrm{RGR}) \cdot t)), \\
& W=\left( \pm W_{\mathbf{0}}\right) \cdot( \pm \exp ((-\mathrm{RGR}) \cdot t)) .
\end{aligned}
$$

Is there a possibility that this mid-existence relates growth function (19) with wave function (20) by bridging the weight gap through operations $\left(0 \pm \boldsymbol{i} W_{\mathbf{0}} \rightarrow( \pm \boldsymbol{i}) \cdot(0\right.$ $\left.\left.\pm \boldsymbol{i} W_{\mathbf{0}}\right)= \pm \boldsymbol{i} 0 \pm W_{0}\right)$ ? Shimojo et al. (2003) tried to form relationships between $\exp (\boldsymbol{i} \cdot t)$ and $\exp (t)$ through the operation of multiplying by $\pm \boldsymbol{i}$. However, I do not know what causes this operation.

(C-4) Another relationship between wave function (20) and growth function (19)

Another relationship between wave function (20) and growth function (19) might occur at $t=0$,

$$
\begin{aligned}
& \Psi=\left( \pm W_{0}\right) \cdot( \pm \exp (( \pm \boldsymbol{i} R G R) \cdot t)), \\
& \rightarrow\left( \pm W_{0}\right) \cdot( \pm \exp (( \pm \boldsymbol{i} R G R) \cdot 0))
\end{aligned}
$$

$$
\begin{aligned}
& = \pm W_{0} \\
& =\left( \pm W_{0}\right) \cdot( \pm \exp (( \pm \mathrm{RGR}) \cdot 0)) \\
& \rightarrow W=\left( \pm W_{0}\right) \cdot( \pm \exp (( \pm \mathrm{RGR}) \cdot t)) .
\end{aligned}
$$

However, I do not know what changes wave functions into growth functions at $t=0$.

$(C-5)$ Combining operations $(C-3)$ and $(C-4)$

Combining operations $(C-3)$ and $(C-4)$ is given, for example, by

$$
\begin{aligned}
& \Psi=\left( \pm W_{0}\right) \cdot( \pm \exp (( \pm \boldsymbol{i} \mathrm{RGR}) \cdot t)) \\
& =\left( \pm W_{0}\right) \cdot( \pm \exp (( \pm \boldsymbol{i}) \cdot(\mathrm{RGR} \cdot t))) \\
& \rightarrow\left( \pm W_{0}\right) \cdot( \pm \exp (( \pm \boldsymbol{i}) \cdot(\pi / 2))) \\
& =0 \pm \boldsymbol{i} W_{0} \\
& \rightarrow( \pm \boldsymbol{i}) \cdot\left(0 \pm \boldsymbol{i} W_{0}\right) \\
& = \pm \boldsymbol{i} \cdot 0 \pm W_{\mathbf{0}} \\
& = \pm W_{\mathbf{0}} \\
& =\left( \pm W_{\mathbf{0}}\right) \cdot( \pm \exp (( \pm \mathrm{RGR}) \cdot 0)) \\
& \rightarrow W_{=}\left( \pm W_{\mathbf{0}}\right) \cdot( \pm \exp (( \pm \mathrm{RGR}) \cdot t)) .
\end{aligned}
$$

This operation suggests bridging the weight gap between $\pm W_{0}$ and $0 \pm \boldsymbol{i} W_{0}$. However, this is only a hypothesis that includes mistakes that I do not notice.

\section{(D) Properties of wave function (20)}

Wave function (20) gives eight functions by the combination of three sets of \pm signs. In this section, each of them will be given its properties with the aid of Euler's formula.

(D-1) $\Psi_{1}=W_{0} \cdot \exp (\boldsymbol{i} \mathrm{RGR} \cdot t)$

$$
=W_{0} \cdot(\cos (\mathrm{RGR} \cdot t)+\boldsymbol{i} \sin (\mathrm{RGR} \cdot t)) .
$$

Function (23) describes an anticlockwise rotation on the complex plane. Three-dimensional (3-D) coordinates (23-1-1), hypothetically suggested from function (23-1), gives a right-handed spiral (Yoshida, 2000),

$$
(\mathrm{RGR} \cdot t, \cos (\mathrm{RGR} \cdot t), \boldsymbol{i} \sin (\mathrm{RGR} \cdot t)) .
$$

$$
\begin{aligned}
(\mathrm{D}-2)-\Psi_{\mathbf{1}} & =\left(-W_{0}\right) \cdot \exp (\boldsymbol{i} \mathrm{RGR} \cdot t) \\
& =\left(-W_{\mathbf{0}}\right) \cdot(\cos (\mathrm{RGR} \cdot t)+\boldsymbol{i} \sin (\mathrm{RGR} \cdot t)) .
\end{aligned}
$$

Function (24) gives a phase shift of $\pi$ from function (23), describing an anticlockwise rotation on the complex plane. Three-D coordinates (24-1-1) suggested from function (24-1) gives a right-handed spiral, 
$(\mathrm{RGR} \cdot t,-\cos (\mathrm{RGR} \cdot t),-\boldsymbol{i} \sin (\mathrm{RGR} \cdot t)) . \quad(24-1-1)$

(D-3) $\Psi_{2}=W_{0} \cdot(-\exp (\boldsymbol{i} \mathrm{RGR} \cdot t))$

$$
=W_{0} \cdot(-\cos (\mathrm{RGR} \cdot t)-\boldsymbol{i} \sin (\mathrm{RGR} \cdot t)) .
$$

Function (25), which is effectively the same as function (24), describes an anticlockwise rotation on the complex plane. Three-D coordinates (25-1-1) suggested from function (25-1) gives a right-handed spiral,

$$
(\mathrm{RGR} \cdot t,-\cos (\mathrm{RGR} \cdot t),-\boldsymbol{i} \sin (\mathrm{RGR} \cdot t)) .
$$

$$
\begin{aligned}
(\mathrm{D}-4)- & \Psi_{2}=\left(-W_{0}\right) \cdot(-\exp (\boldsymbol{i} \mathrm{RGR} \cdot t)) \\
& =\left(-W_{\mathbf{0}}\right) \cdot(-\cos (\mathrm{RGR} \cdot t)-\boldsymbol{i} \sin (\mathrm{RGR} \cdot t)) .
\end{aligned}
$$

Function (26), which is effectively the same as function (23), describes an anticlockwise rotation on the complex plane. Three-D coordinates (26-1-1) suggested from function (26-1) gives a right-handed spiral,

$(\mathrm{RGR} \cdot t, \cos (\mathrm{RGR} \cdot t), \boldsymbol{i} \sin (\mathrm{RGR} \cdot t))$.

$$
(\mathrm{D}-5) \quad \Psi_{3}=W_{0} \cdot \exp ((-\boldsymbol{i} \mathrm{RGR} \cdot t))
$$

$$
\begin{aligned}
& =W_{\mathbf{0}} \cdot\left(\cos ((-\mathrm{RGR}) \cdot t)+\boldsymbol{i}_{\mathrm{sin}}((-\mathrm{RGR}) \cdot t)\right),(27-1) \\
& =W_{\mathbf{0}} \cdot\left(\cos (\mathrm{RGR} \cdot t)-\boldsymbol{i}_{\sin }(\mathrm{RGR} \cdot t)\right) .
\end{aligned}
$$

Function (27) describes a clockwise rotation on the complex plane. Three-D coordinates (27-1-1) suggested from function (27-1) gives a right-handed spiral, but $3-\mathrm{D}$ coordinates (27-2-1) suggested from function (27-2) gives a left-handed spiral,

$$
((-\mathrm{RGR} \cdot t), \cos ((-\mathrm{RGR}) \cdot t), \boldsymbol{i} \sin ((-\mathrm{RGR}) \cdot t)),
$$

$(\mathrm{RGR} \cdot t, \cos (\mathrm{RGR} \cdot t),-\boldsymbol{i} \sin (\mathrm{RGR} \cdot t))$

I do not know why this inverse phenomenon occurs when the same function is described using another way.

$$
\begin{aligned}
(\mathrm{D}-6)- & \Psi_{3}=\left(-W_{0}\right) \cdot \exp ((-\boldsymbol{i} \mathrm{RGR}) \cdot t) \\
& =\left(-W_{0}\right) \cdot(\cos ((-\mathrm{RGR}) \cdot t)+\boldsymbol{i} \sin ((-\mathrm{RGR}) \cdot t)) \\
& =\left(-W_{0}\right) \cdot\left(\cos (\mathrm{RGR} \cdot t)-\boldsymbol{i}_{\sin }(\mathrm{RGR} \cdot t)\right)
\end{aligned}
$$

Function (28) gives a phase shift of $\pi$ from function (27), describing a clockwise rotation on the complex plane. Three-D coordinates (28-1-1) suggested from function (28-1) gives a right-handed spiral, but 3-D coordinates (28-2-1) suggested from function (28-2) gives a lefthanded spiral,

$$
\begin{array}{lr}
((-\mathrm{RGR} \cdot t),-\cos ((-\mathrm{RGR}) \cdot t),-\boldsymbol{i} \sin ((-\mathrm{RGR}) \cdot t)), \\
(28-1-1) \\
(\mathrm{RGR} \cdot t,-\cos (\mathrm{RGR} \cdot t), \boldsymbol{i} \sin (\mathrm{RGR} \cdot t)) . & (28-2-1)
\end{array}
$$

(D-7) $\Psi_{4}=W_{0} \cdot(-\exp ((-\boldsymbol{i} \mathrm{RGR}) \cdot t))$

$$
=W_{0} \cdot\left(-\cos ((-\mathrm{RGR}) \cdot t)-\boldsymbol{i}_{\sin }((-\mathrm{RGR}) \cdot t)\right) \text {, }
$$

$$
=W_{0} \cdot(-\cos (\mathrm{RGR} \cdot t)+\boldsymbol{i} \sin (\mathrm{RGR} \cdot t)) .
$$

Function (29), which is effectively the same as function (28), describes a clockwise rotation on the complex plane. Three-D coordinates (29-1-1) suggested from function (29-1) gives a right-handed spiral, but 3-D coordinates (29-2-1) suggested from function (29-2) gives a left-handed spiral,

$$
((-\mathrm{RGR} \cdot t),-\cos ((-\mathrm{RGR}) \cdot t),-\boldsymbol{i} \sin ((-\mathrm{RGR}) \cdot t)),
$$

$$
(\mathrm{RGR} \cdot t,-\cos (\mathrm{RGR} \cdot t), \boldsymbol{i} \sin (\mathrm{RGR} \cdot t)) .
$$

$(\mathrm{D}-8)-\Psi_{4}=\left(-W_{0}\right) \cdot(-\exp ((-\boldsymbol{i} \mathrm{RGR}) \cdot t))$,

$$
=\left(-W_{0}\right) \cdot\left(-\cos ((-\mathrm{RGR}) \cdot t)-\boldsymbol{i}_{\sin }((-\mathrm{RGR}) \cdot t)\right),
$$

$$
=\left(-W_{0}\right) \cdot(-\cos (\operatorname{RGR} \cdot t)+\boldsymbol{i} \sin (\operatorname{RGR} \cdot t)) .
$$

Function (30), which is effectively the same as function (27), describes a clockwise rotation on the complex plane. Three-D coordinates (30-1-1) suggested from function (30-1) gives a right-handed spiral, but 3-D coordinates (30-2-1) suggested from function (30-2) gives a left-handed spiral,

$$
((-\mathrm{RGR} \cdot t), \cos ((-\mathrm{RGR}) \cdot t), \boldsymbol{i} \sin ((-\mathrm{RGR}) \cdot t)),
$$

$(\mathrm{RGR} \cdot t, \cos (\mathrm{RGR} \cdot t),-\boldsymbol{i} \sin (\mathrm{RGR} \cdot t))$.

$(30-2-1)$

\section{(E) Rotations and spirals in wave function}

\section{(E-1) Rotations}

As shown in section (D), a group of four wave functions (23) (26) give anticlockwise rotations and the other group of four wave functions (27) (30) give clockwise rotations. In each group, two functions show a phase shift of $\pi$ from the other two functions. There are eight wave functions apparently, but actually there are four wave functions when duplication is avoided. (E-2) Spirals

Three-D coordinates have been suggested hypothetically from each of eight wave functions by transforming them into trigonometric functions according to Euler's formula (Yoshida, 2000). Eight coordinates (23-1-1) (30-1-1) suggested from wave functions (23-1) (30-1) give right-handed spirals. Each of four wave functions (27-1) (30-1) has another way of description: wave functions (27-2) (30-2). However, four coordinates (27-2-1) (30-2-1) suggested from wave functions (27-2) (30-2) give left-handed spirals, inversely winded spirals in contrast to right-handed spirals given by coordinates (27-1-1) (30-1-1) that are 
suggested from wave functions (27-1) (30-1). Thus, the number of right-handed spirals is eight and that of left-handed spirals is four, where the latter spirals are derived from half of the former spirals as a result of another way of description of wave function. I do not know why 3-D coordinates suggested from wave functions basically show right-handed spirals, and why lefthanded spirals are derived when wave functions are given another way of description. There are eight wave functions apparently, but actually there are four wave functions when duplication is avoided.

\section{(F) Problems}

There is a kind of unnaturalness in the present study because of forcedly deriving wave functions of complex number from a differential equation for growth functions of the ruminant animal and the forage plant. This deriving is not conducted when the differential equation is solved, because growth phenomena are not related to wave phenomena. However, one of the viewpoints that I have done this work is that both functions are described using the exponential function with base $e$ (Napier's number). This is the only common feature between the two functions that are absolutely different. The hypothesis that I have is that wave functions of complex number might exist at the back of growth functions. The objective of the present study was to approach this difficult problem, but actually this resulted in suggesting doubtful hypotheses to the comparison and relationship between wave functions and growth functions. New ideas are required in order to approach this problem again.

\section{(G) Conclusions}

It is suggested from the present study that introducing growth jerk into basic growth mechanics gives some pieces of information about properties of wave functions in the comparison and relationship with growth functions.

\section{REFERENCES}

Shimojo, M., K. Ikeda, Y. Asano, R. Ishiwaka, T. Shao, H. Sato, M. Tobisa, Y. Nakano, N. Ohba, Y. Yano and Y. Masuda 2003 Exponential functions with base $e$ in growth analysis and deriving them from rotations of axes of time described using Euler's formula. J. Fac. Agr., Kyushu Univ., 48: 65-69

Shimojo, M. 2006 Introducing viewpoints of mechanics into basic growth analysis - (II) Relative growth rate compared with energy in wave function -. J. Fac. Agr., Kyushu Univ., 51 $289-291$

Shimojo, M. 2007 Introducing viewpoints of mechanics into basic growth analysis - (V) Problems derived from introducing growth jerk -. J. Fac. Agr., Kyushu Univ., 52: 77-79

Shimojo, M., T. Shao and Y. Masuda 2008 Introducing viewpoints of mechanics into basic growth analysis - (VII) Mathematical properties of basic growth mechanics in ruminant -. J. Fac. Agr., Kyushu Univ., 53: 453-458

Yoshida, T. 2000 Emotion for imaginary number. Tokai University Press, Tokyo. (written in Japanese) 